\title{
Helping students understand optical concepts by development of digital holographic teaching apparatus
}

Dayong Wang, Bin Li, Yunxin Wang, Lu Rong, Jie Zhao

Dayong Wang, Bin Li, Yunxin Wang, Lu Rong, Jie Zhao, "Helping students understand optical concepts by development of digital holographic teaching apparatus," Proc. SPIE 9793, Education and Training in Optics and

Photonics: ETOP 2015, 979335 (8 October 2015); doi: 10.1117/12.2230022

Event: Education and Training in Optics and Photonics: ETOP 2015, 2015, Bordeaux, France 


\title{
Helping students understand optical concepts by development of digital holographic teaching apparatus
}

\author{
Dayong Wang ${ }^{\text {a }}$, Bin $\mathrm{Li}^{\mathrm{a}}$, Yunxin Wang* a ${ }^{*}$ Lu Rong ${ }^{\mathrm{a}}$, Jie Zhao ${ }^{\mathrm{b}}$

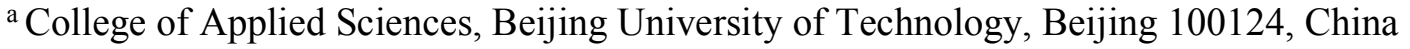 \\ $\mathrm{b}$ The Pilot College, Beijing University of Technology, Beijing 101101, China
}

\begin{abstract}
Lightwave, as a kind of electromagnetic wave, possesses a frequency much higher than the response frequency of the current photodetectors, so the complex amplitude of the lightwave cannot be measured directly due to the loss of the phase information. Some invisible optical concepts such as phase, wavefront etc. are difficult to understand for the undergraduate students. To make the wavefront of the light wave visible, a digital holographic teaching apparatus, as a kind of comprehensive experimental platform, is developed by our teachers and students under the support of Daheng Science \&Technology company. Digital holography (DH) can retrieve the quantitative amplitude and phase information of the object wavefront, which makes the wavefront display possible. In the teaching apparatus, the digital hologram is recorded by the off-axis lensless Fourier transform (LFT) digital holographic system, and the reconstruction of the hologram is achieved by the inverse Fourier Transform. The system is controlled by the Labview software. Except the quantitative amplitude and phase information, the spectrum of the hologram in the spatial-frequency domain etc. can also be visualized in the user interface of the apparatus. This can help students intuitively comprehend many optical content including phase, optical interference, diffraction, optical Fourier transform and diffraction propagation calculation et al., which has the characteristics of being open, flexible and systematic. Furthermore, the analytical and manipulative ability of students can be effectively advanced, and students can also generally understand the actual demand, work pattern and product sales of the company.
\end{abstract}

Keywords: Optical concept; Digital holography; Wavefront; Teaching apparatus

\section{INTRODUCTION}

The frequency of Light-wave is much higher than the response frequency of the current photodetector due to which it is not possible presently to directly measure the complex amplitude of the light wave, and it results in the loss of the phase information. To make the wavefront of the light wave visible, a digital holographic teaching apparatus as a kind of comprehensive experimental platform is developed. Digital holography (DH) can retrieve the quantitative amplitude and phase information of the object wavefront. In 1948, Dennis Gabor put forward the holography for the improvement of the resolution of electron microscope ${ }^{[1]}$. The basic principle of holography is as follows: firstly, the object and the reference wave are made to interfere at the recorded plate, where these interference fringes are called hologram and include the amplitude and phase information of the object. Secondly, the hologram is reconstructed under illumination by the same reference wave, and the reconstructed image can be produced due to the diffraction of the hologram. In 1967, Goodman and Lawrence put forward the concept of digital holography ${ }^{[2]}$. Digital holography ${ }^{[3-7]}$ is actually a coherent optical imaging system where the electronic imaging instrument (such as CCD) is used for the recording of hologram instead of the photosensitive plate. The reconstruction was performed by the numerical calculation. With the advance of science and technology, the digital holography has been applied in many fields, such as temperature measurement ${ }^{[8]}$, living cells imaging ${ }^{[9]}$, particle field measurement ${ }^{[10]}$, refractive index determination ${ }^{[11]}$ and so on.

In this paper a setup of lensless Fourier transform digital holographic system is built as a kind of comprehensive experimental platform under the support of Daheng Science \& Technology company. The lensless Fourier transform digital holographic system is very simple ${ }^{[\mathbf{1 2}]}$, and the reconstructed image can easily be obtained by the Fourier transform algorithm. In order to understand the concept clearly by observing the hologram reconstruction process, we programmed the process of the reconstruction by the Labview software. The Labview software can show us the quantitative amplitude and phase information of the object. The thicker optical elements can bring some phase wrap in

Education and Training in Optics and Photonics: ETOP 2015, edited by Eric Cormier, Laurent Sarger Proc. of SPIE Vol. 9793, 979335 - @ 2015 SPIE, IEEE, OSA, ICO · doi: 10.1117/12.2230022 
the reconstructed phase image, so the phase unwrapping is put into the software. After the process of phase unwrapping, the reconstructed phase image reveals the true phase information of the object correctly. The designed apparatus is helpful for the undergraduate students to understand the optical principle including phase, optical interference, diffraction and diffraction propagation calculation et al.

\section{METHODOLOGY}

The spatial frequency of lensless Fourier transform digital holographic interference fringes is almost evenly on detecting plane ${ }^{[13,14]}$, so the lensless Fourier transform digital holography easier fulfill the sampling conditions. The shorter is the record distance, the higher will be the resolution of the reconstructed image. The schematic diagram of lensless Fourier transform digital holography is shown in Fig. 1. Here $\left(x_{0}, y_{0}\right)$ and $(x, y)$ represents the Cartesian coordinate system of the object plane and the hologram plane respectively, and $t\left(x_{0}, y_{0}\right)$ is the complex amplitude transmittance of the object wave. It should be noted that the reference point source and object are in the same plane. For the location of the reference point source, the distance of the $x_{0}$ direction is $-a$ and the distance of the $y_{0}$ direction is $-b$. The distance between the object plane and the CCD plane is $d$.

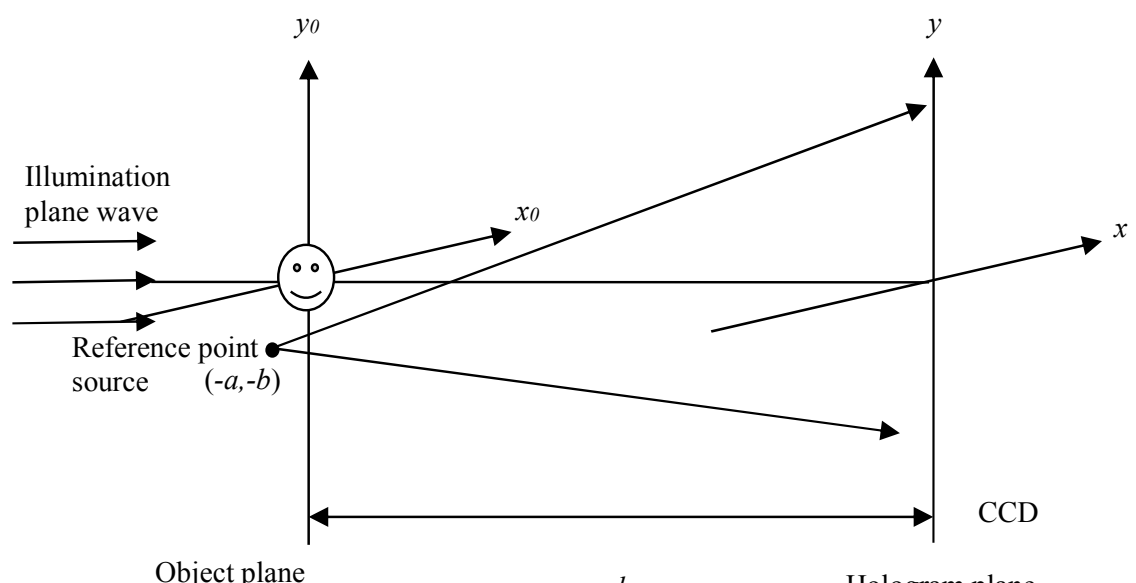

Fig. 1 Schematic diagram of Lensless Fourier transform digital holography

A plane wave of the unit amplitude is used to illuminate the object. The distribution of the object wave on the CCD plane is given with the Fresnel diffraction integral formula, as follows:

$$
O(x, y)=\exp \left[j \frac{k}{2 d}\left(x^{2}+y^{2}\right)\right] \iint_{-\infty}^{\infty} t\left(x_{0}, y_{0}\right) \exp \left[j \frac{k}{2 d}\left(x_{0}^{2}+y_{0}^{2}\right)\right] \times \exp \left[-j \frac{2 \pi}{\lambda d}\left(x x_{0}+y y_{0}\right)\right] d x_{0} d y_{0} .
$$

Here, in order to simplify the Eq.(1), it is denoted that

$$
t^{\prime}\left(x_{0}, y_{0}\right)=t\left(x_{0}, y_{0}\right) \exp \left[j \frac{k}{2 d}\left(x_{0}^{2}+y_{0}^{2}\right)\right]
$$

and

$$
T^{\prime}\left(\frac{x}{l d}, \frac{y}{l d}\right)=\mathrm{FT}\left\{t^{\prime}\left(x_{0}, y_{0}\right)\right\}
$$

where FT \{\} represents the Fourier transform operation. Then Eq. (1) can be represented by 


$$
O(x, y)=\exp \left[j \frac{k}{2 d}\left(x^{2}+y^{2}\right)\right] \mathrm{T}^{\prime}\left(\frac{x}{\lambda d}, \frac{y}{\lambda d}\right) .
$$

The reference wave is a spherical wave and its distribution on the CCD plane is given as

$$
R(x, y)=\exp \left[j \frac{k}{2 d}\left(x^{2}+y^{2}\right)\right] \exp \left[j \frac{2 \pi}{\lambda d}(a x+b y)\right] .
$$

The reference wave and the object wave interfere on the CCD plane, where the distribution of hologram is

$$
I(x, y)=|O(x, y)|^{2}+|R(x, y)|^{2}+O(x, y) R^{*}(x, y)+O *(x, y) R(x, y)
$$

The hologram is recorded and digitized by the CCD as the digital image in the computer. It is easy to be stored and sent through the network. Also the reconstruction will be done in the computer and exhibits the great flexibility.

Next the reconstruction will be discussed. There are three parts in Eq. (6), where both $|O(x, y)|^{2}$ and $|R(x, y)|^{2}$ together represent the first part named the zero order, and $O(x, y) R^{*}(x, y)$ and $O^{*}(x, y) R(x, y)$ are the second and third parts, which will produce the real image and the conjugate image separately.

To focus on the real image, only the second part of the reconstructed image is analyzed that is $O(x, y) R *(x, y)$ as given below

$$
O(x, y) R^{*}(x, y)=\exp \left[-j \frac{2 \pi}{\lambda d}(a x+b y)\right] \mathrm{T}^{\prime}\left(\frac{x}{\lambda d}, \frac{y}{\lambda d}\right)
$$

The digital hologram is reconstructed by the inverse Fourier transform, where $f_{x}=x / \lambda d$ and $f_{y}=y / \lambda d$. The reconstructed image is

$$
\begin{aligned}
U_{1}\left(x_{i}^{\prime}, y_{i}^{\prime}\right) & =\iint_{-\infty}^{\infty} T^{\prime}\left(\frac{x}{\lambda d}, \frac{y}{\lambda d}\right) \exp \left[-j \frac{2 \pi}{\lambda d}(a x+b y)\right] \exp \left[-j \frac{2 \pi}{\lambda d}\left(x_{i}^{\prime} x+y_{i}^{\prime} y\right)\right] d x d y \\
& =(\lambda d)^{2} t^{\prime}\left(-x_{i}^{\prime},-y_{i}^{\prime}\right)^{*} \delta\left(-x_{i}^{\prime}-a,-y_{i}^{\prime}-b\right)
\end{aligned}
$$

The original coordinate is shifted by 180 degrees and the new coordinate is $\left(x_{i}, y_{i}\right)$ and the reconstructed image is obtained by substituting Eq. (2) into Eq. (8) as given below

$$
U_{1}\left(x_{i}, y_{i}\right)=(\lambda d)^{2} t\left(x_{i}-a, y_{i}-b\right) \exp \left\{j \frac{k}{2 d}\left[\left(x_{i}-a\right)^{2}+\left(y_{i}-b\right)^{2}\right]\right\}
$$

Therefore, from Eq. (9) the amplitude reconstruction result is the accurate information of the object at the center $(a, b)$. For the phase distribution, unfortunately the quadratic phase factor will result in the phase aberration, which is closely related to the recording distance and the location of the reference wave. However, these parameters are difficult to be measured accurately in experiments. So it is necessary to deal with the phase distortion with the methods such as digital mask, and the double exposure. Finally, it is worth to note that the numerical case of FFT needs meet the constraint of optical propagation, and the pixel interval of the reconstruction of image plane is $\lambda d / N \Delta x$, where $N$ and $\Delta x$ are the pixel number and pixel size of the CCD, respectively.

\section{EXPERIMENTS}

A setup of lensless Fourier transform digital holographic system is built with the usual optical products. The schematic of the system is illustrated in Fig. 2, which is based on the Mach-Zehnder interferometer. The wavelength of the laser is $532 \mathrm{~nm}$, and it passes through the neutral filter (NF) and the polarization beam splitter(PBS). The NF can regulate the intensity of the laser. Then the laser beam is divided into two waves by the PBS. One wave goes through the transparent or semi-transparent test sample as the object wave, while the other wave is expanded and filtered to produce a finer reference point source. The object wave and the reference wave are combined again by the beam splitter (BS), then the interference pattern is recorded by the $\mathrm{CCD}$ detector. The intensity ratio between the reference and object beams can be 
adjusted by the combination of $\lambda / 2$ half-wave plate $\left(\mathrm{HP}_{1}\right.$ and $\left.\mathrm{HP}_{2}\right)$ and $\mathrm{PBS}$ to improve the fringe quality of the digital hologram. The lensless Fourier transform holography requires that the object and the reference point source should be at the same distance from the CCD. In the experiment we used a one-dimensional grating as object which has period of $300 \mu \mathrm{m}$ and the depth of $1 \mu \mathrm{m}$. The lensless Fourier transform digital holographic experiment setup is shown in Fig. 3.

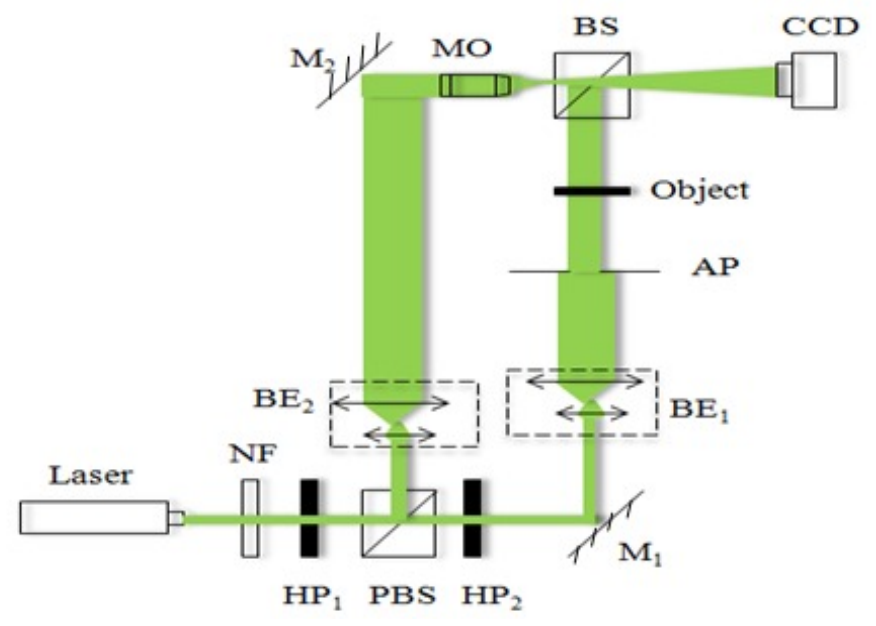

Fig. 2 The schematic of the lensless Fourier digital holography
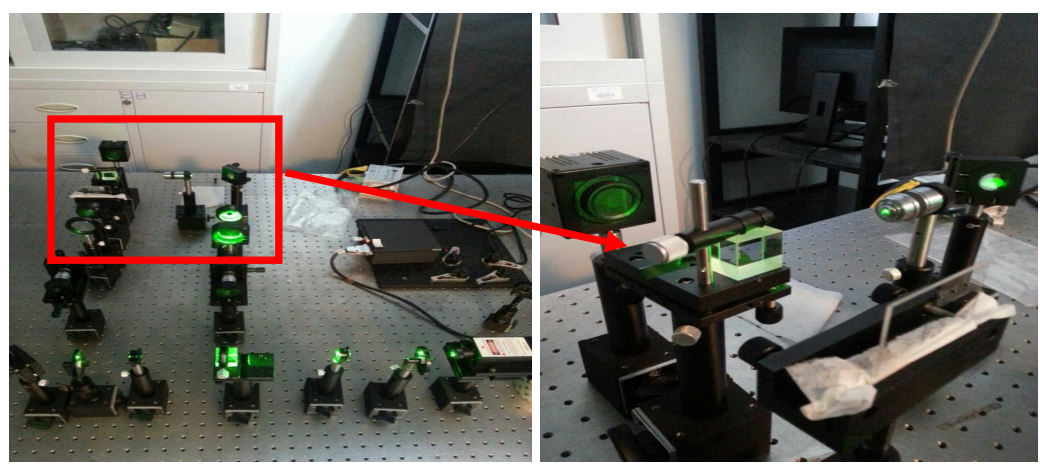

Fig. 3 Lensless Fourier digital holographic experiment setup

The advantages of the Labview software are the visualization and integration. The interface of the lensless Fourier transform based on Labview software is shown in Fig. 4. In the case of the optical path larger than one wavelength, the reconstructed phase image has the wrapped phase problem due to the $2 \pi$ period of the phase information. The reconstructed phase image needs the process of the phase unwrapping. The results of lensless Fourier transform digital holography is shown in Fig. 5, where Fig. 5(a), (b) and (c) are the digital hologram, its reconstructed phase image and the three dimensional view of reconstructed phase image, respectively.

With the system, the undergraduate students can adjust the experiment setup themselves and change the object such as the micro-lens array, biological cells and so on. If the undergraduate students have more interests, it is also possible for them to measure the reflective object by rearranging the experiment setup. 


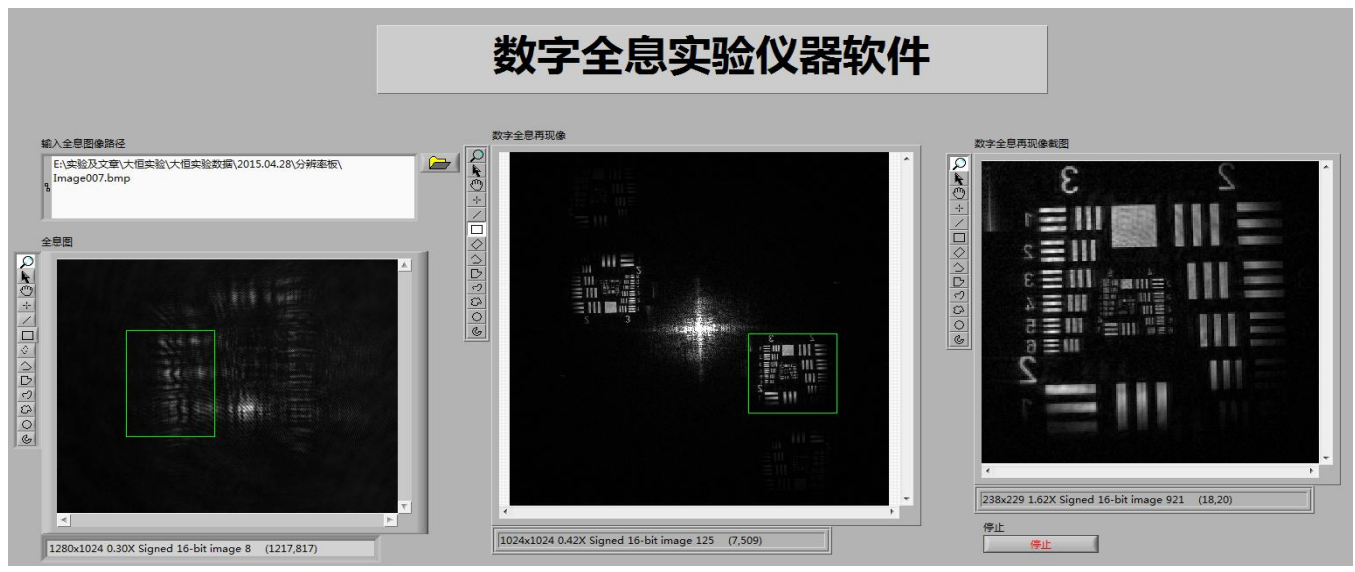

Fig 4. The interface of the lensless Fourier transform holographic system based on Labview software

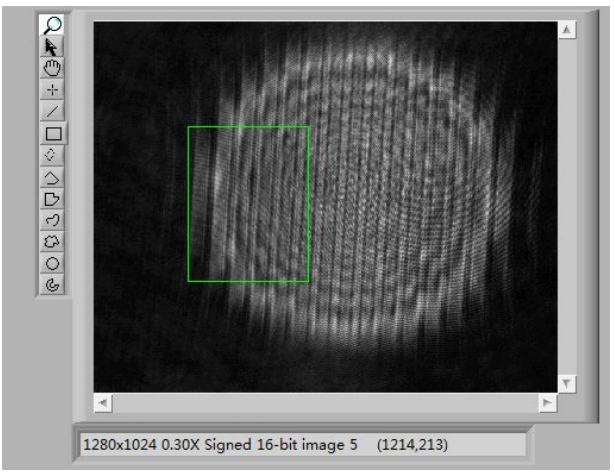

(a) digital hologram

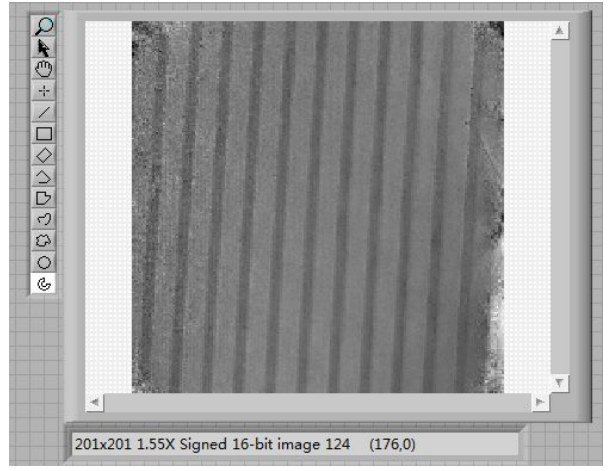

(b) reconstructed phase image

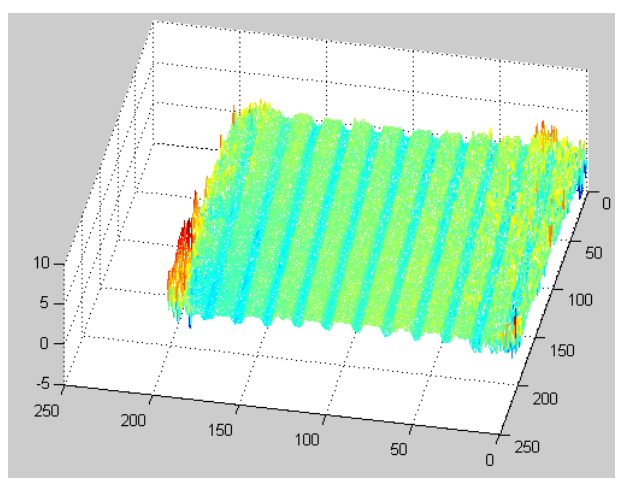

(c) three dimensional view of (b)

Fig. 5 The experimental results of Lensless Fourier transform digital holographic imaging system;

(a) digital hologram, (b) reconstructed phase image, and (c) three dimensional view of (b).

\section{CONCLUSIONS}

The comprehensive teaching platform is introduced for the better understanding of optical concepts based on the digital holographic technology. Digital holography can get the quantitative phase and amplitude information of the object. The process of recording and reconstruction of the hologram is presented theoretically. Then the process of recording and the reconstruction of hologram is analyzed and included into the comprehensive experimental platform. In the aspect of 
numerical calculation, each step can be visualized for the hologram reconstruction by the Labview software. As the phase image has the problem of phase wrapping, the phase image needs the process of the phase unwrapping. Finally we can get the true phase information of the object. With the experimental platform, it can help students intuitively comprehend many optical content including phase, optical interference, diffraction, optical Fourier transform and diffraction propagation calculation et al., The system also has the characteristics of being open, flexible and systematic.

\section{ACKNOWLEDGMENTS}

This work is financially supported by the National Natural Science Foundation of China (No. 61307010 and 61205010), the Beijing Municipal Natural Science Foundation (No. 1122004), the Research Fund for the Doctoral Program of Higher Education of China (No.20121103120003), and the Beijing University Fund for Scientific Research of Doctor (No. X0006111201102), Graduate Technology Fund of Beijing University of Technology under Grant No. ykj-20139357.

\section{REFERENCES}

[1] D. Gabor, "A new microscopic principle," Nature, 161 (4098), 777-778 (1948).

[2] J. W. Goodman, R. W. Lawrence, "Digital image formation from electronically detected holograms," Appl. Phys. Lett., 11 (3) , 77-79 (1967).

[3] U. Schnars and W. Jüptner, "Direct recording of holograms by a CCD target and numerical reconstruction," Appl. Opt., 33(2), 179-181 (1994).

[4] E. Cuche, F. Bevilacqua, and C. Depeursinge, "Digital holography for quantitative phase-contrast imaging," Opt. Lett., 24(5), 191-193 (1999).

[5] G. Pedrini, S. Schedin and H. Tiziani, "Lensless digital holographic interferometry for the measurement of large objects," Opt. Commun., 171(3), 29-36 (1999).

[6] S. Grilli, P. Ferraro, S. De Nicola, A. Finizio, G. Pierattini, and R. Meucci, "Whole optical wavefields reconstruction by digital holography," Opt. Exp., 9(6), 294-302 (2001).

[7] Y. Zhang, Q. Lü, B. Ge, H. Zhao, and Y. Sun, "Digital holography and its application," Proc. SPIE 5636, $200-211$ (2005).

[8] S. Sharma, G. Sheoran, C. Shakher, "Temperature measurement of axisymmetric flame under the influence of magnetic field using Lensless Fourier transform digital holography," Appl. Opt., 51(19), 4554-4562 (2012).

[9] Y. Wang, D. Wang, J. Zhao, et al., "Non-invasive monitoring of living cell culture by lensless digital holography imaging," Chin. Opt. Lett., 9(3), 030901-1 - 4 (2011).

[10] F. Dubois, N. Callens, C. Yourassowsky, et al., " Digital holographic microscopy with reduced spatial coherence for three-dimensional particle flow analysis," Appl. Opt., 45(5), 864-871 (2006).

[11] M. M. Hossain, D. S. Mehta, C. Shakher, "Refractive index determination: an application of Lensless Fourier digital holography," Opt. Eng., 45(10), 106203-1 - 7 (2006).

[12] D. Dirksen, H. Droste, B. Kemper, et al., "Lensless Fourier holography for digital holographic interferometry on biological samples," Opt. and Las. in Eng., 36 (3) , 241-249 (2001).

[13] M. Gustafsson, M. Sebesta, B. Bengtsson, et al., "High-resolution digital transmission microscopy a Fourier holography approach," Opt. \& Las .Eng., 41(2), 553-563 (2004).

[14] I. Banyasz, J. Kornis, "High-resolution lensless Fourier-transform digital holography," Proc. SPIE 5856, $71-79$ (2005). 